\title{
Nonlinear Vibrations and Buckling Solution for the Uniform Hinged Beam
}

\author{
Gondi Konda Reddy \\ Associate Professor, Mechanical Engineering Department, Sreenidhi Institute of Science and Technology, \\ Ghatkesar, Hyderabad, Telangana state, INDIA-501301.
}

\begin{abstract}
Some improvisations, to obtain the closed form nonlinear Eigen solutions, for example solutions for nonlinear free vibrations and nonlinear buckling problems of a hinged beam, which can be solved by following standard and classical energy methods, are proposed. These improvisations simplify the solution procedure of said problem significantly, by providing a logical approach to determine the functional form for the admissible function for the axial displacement, by reducing the number of undetermined coefficients from twolfor beam problems with single term admissible to function) one, which is achieved by determining the undetermined coefficient of the axial displacement in terms of the undetermined coefficient of the lateral displacement and finally by a method of converting the solution of the nonlinear free vibration problem with harmonic oscillations assumed to a rigorous solution without this assumption. The solutions obtained in a simple way by using the improvisations mentioned match exactly with the respective classical solutions for the Eigen problems mentioned above. The improvisations used here are general with no assumptions and hence can be used for obtaining the solutions of the more complex Eigen problems mentioned for other commonly used structural elements in a much simpler way with the same accuracy.
\end{abstract}

Keywords: Nonlinear vibrations, nonlinear buckling, hinged-hinged beam, Strain displacement relations

\section{Introduction}

Light weight thin walled beams used in modern complex structures are prone to undergo large deformations because of their high flexibility. The Eigen behaviour of such beams in nonlinear and this effect(geometric nonlinearity) has to be included in studying the nonlinear (large amplitude) free vibration and the nonlinear buckling(post buckling) behaviour with reference to the amplitude / deflection at a given point of the beam.

The uniform, slender hinged-hinged beam with axially immovable ends, which means the axial displacements are zero at the ends of the beam, is a bench mark problem, and many researchers consider this configuration of the beam to validate their new formulations/results.

The Eigen solutions considered in the paper are

a) The nonlinear free vibration and b) the post buckling behaviour of hinged beams.

The first classical paper on the nonlinear free vibrations of hinged beams is due to Woinowsky-Krieger[1]. Since then many papers appeared in the open literature, considering the same beam with different formulations using both continuum and numerical methods[2]. These references are not an exhaustive list and one may see Ref. [3] for a better understanding of the various formulations and also to have near complete list of references on this topic.

Compared to the nonlinear free vibration problem, the literature available on the post buckling problem is limited. Some of the prominent books on this field are due to Dym[4],Thamson and Hunt[5] etc. considered the problem. For post buckling problem it is evident that for the beam configuration considered here does not allow the externally applied axial concentrated compressive load to penetrate the hinged ends, because of the axially immovable conditions considered at the ends, and as a result the beam does not feel the effect of the applied load at the ends.

However, one can imagine a situation that when the beam is subjected to a uniform heating above the ambient temperature, a compressive load will be generated and the study of the nonlinear buckling behaviour of the beam, which is generally called the thermal nonlinear buckling, which occurs when the beam experiences the externally applied axial compressive load at the ends, when the ends are free to move axially. It is to be noted here that the nonlinearity involved in the study of the nonlinear thermal buckling, the pioneering work on this topic can be seen in Ref.[6].The steady state response of free and forced vibration of beams with restrained ends are explained in the Ref. [7].It was discussed variational iteration method in the Ref[8] is complicated mathematical analysis. Similar kind of analysis also observed in Ref $[9,10]$. 
For the beam configuration considered here the nonlinearity involved is in the strain-displacement relations(von Karman type), and is due to the axially immovable and conditions of the beam. Further, a relation between the compressive load developed in the beam because of uniform heating can be easily obtained and is given later for completeness.

\section{Nonlinear Vibrations}

The stain-displacement relations of a beam undergoing large amplitudes are (Fig.1)

$$
\begin{aligned}
& \varepsilon_{x}=u_{x}+\frac{1}{2} w_{x}^{2} \\
& \text { And } \Psi_{x}=-w_{x x}
\end{aligned}
$$

Where ' $\varepsilon_{x}$ ' and ' $\Psi_{x}$ ' are the axial strain and curvature, ' $u$ ' is the axial displacement, $w$ is the lateral displacement, ' $x$ ' is the axial coordinate and suffix ' $x$ ' denotes differentiation with respect to ' $x$ '.

The strain energy ' $U$ ' of the beam of length ' $L$ ' is given

$$
U=\frac{E A}{2} \int_{0}^{L}\left[u_{x}{ }^{2}+u_{x} w_{x}{ }^{2}+\frac{1}{4} w_{x}{ }^{4}\right] d x+\frac{E I}{2} \int_{0}^{L} w_{x x}{ }^{2} d x
$$

The admissible functions chosen for $\mathrm{W}$

$$
W=b \sin \frac{\pi x}{L}
$$

The admissible function for $\mathrm{u}$ can be calculated from the formula.

$$
\frac{d^{2} u}{d x^{2}}=-\frac{d w}{d x} \frac{d^{2} w}{d x^{2}}
$$

From Eq. (5), the admissible function $u$ is

$$
u=\frac{-b^{2} \pi}{8 L} \sin \frac{2 \pi x}{L}
$$

Now, Eqs.(4) and (6) are the admissible functions for ' $u$ ' and ' $w$ ' for the hinged beam and contain only one undetermined coefficient ' $b$ ' Assuming harmonic oscillations, which makes the problem simpler.

The Kinetic energy ' $T$ ' of the vibrating beam is

$$
T=\frac{-m \omega^{2} N L}{2} \int_{0}^{L} w^{2} d x
$$

where, $m$ is the mass per unit length and $\omega_{N L}$ is the nonlinear radian frequency of the beam. Minimizing the Langrangian $(U-T)$ with respect ' $\mathrm{b}$ ', as

$$
\frac{d}{d b}(U-T)=0
$$

Substituting the admissible function for ' $u$ ' and ' $w$ ' in ' $U$ ' and $T$, and from Eq.(8), and after simplification, noting that the linear radian frequency parameter ' $\omega^{2}{ }_{L}{ }^{\prime} i s\left(\pi^{4} E I\right) /\left(m L^{4}\right)$, the ratio of the nonlinear to linear radian frequency of the hinged beam is obtained as

$$
\left(\omega_{N L} / \omega_{L}\right)^{2}=\left\lfloor\left(1+1 / 4(b / r)^{2}\right)\right\rfloor
$$

Where $r$ is the radius of gyration of the beam cross-section. Derivation of the expression for $\left(\omega_{N L} / \omega_{L}\right)^{2}$, using the harmonic oscillations assumption, as given in Eq.(9) is much simpler. Stating with this expression, it is necessary to evaluate the same, when the assumption of harmonic oscillation assumption is not valid, as in the nonlinear free vibrations. For this purpose, we apply the harmonic balance method[7], the brief details of which are given below:

For the nonlinear vibrations of beams, the governing dynamic equation of equilibrium is of the form

$$
q=\alpha_{1} q+\alpha_{2} q^{3}
$$

Where $q$ is the non-dimensional maximum lateral displacement $(=b / r), \quad \alpha_{1}$ and $\alpha_{2}$ are the constants depending on the boundary conditions of the beam, which can be evaluated from the assumed admissible functions and $(\cdot)$ denotes derivation with respect to time ' $t$ '. 
If the cubic term in Eq. (10) is neglected Eq. (10) gets degenerated into the classical linear vibration problem of beams, the radian linear frequency ' $\omega$ ' of which can be easily evaluated as $\omega_{L}=\sqrt{\alpha_{1}}$ Now, the ratio of the nonlinear to the linear radian frequency can be obtained with the harmonic oscillations assumption.

$$
\begin{aligned}
& q=-q \sin \omega_{N L} t \\
& \text { as } \\
& \left(\frac{\omega_{N L}}{\omega_{L}}\right)=\left[1+\frac{\alpha_{2}}{\alpha_{1}}\right] q^{2}
\end{aligned}
$$

Equations (9) and (12) are of the same form with the values for $\alpha_{2} / \alpha_{1}=1 / 4$ and $q=b / r$. The expression for $\omega^{2}{ }_{N L} / \omega^{2}{ }_{L}$ is now derived in a rigorous way by assuming the time variation of $q$ as

$$
q=q_{m} \sin \omega_{N L} t
$$

From Equation (13) and (10), we get

$$
-\omega^{2}{ }_{N L} q_{m} \sin \omega_{N L} t=\alpha_{1} q_{m} \sin \omega_{N L} t+\alpha_{2} q^{3}{ }_{m} \sin ^{3} \omega_{N L} t
$$

Neglecting the higher harmonics, as

$$
\operatorname{Sin}^{3} \omega_{N L} t=3 / 4 \sin \omega_{N L} t
$$

Equations (15) and (14), give the rigorous expression for $\omega^{2}{ }_{N L} / \omega^{2}{ }_{L}$, after simplifications as

$$
\left(\frac{\omega_{N L}}{\omega_{L}}\right)^{2}=\left[1+\frac{3}{4} \frac{\alpha_{2}}{\alpha_{1}}\left(\frac{b}{r}\right)^{2}\right]
$$

Since $q_{m}=b / r$, by definition and $\left(\alpha_{2} / \alpha_{1}\right)=1 / 4$ for the hinged beam. By comparing the equations (9) and (16), it can be concluded that the rigorous expressions for $\left(\omega_{N L} / \omega_{L}\right)^{2}$ can be obtained from the same obtained by the harmonic oscillations assumption by multiplying, the coefficient $(b / r)^{2}$ by a factor $3 / 4$. The expression for $\left(\omega_{N L} / \omega_{L}\right)^{2}$ given in equation (9) is the standard one and is reported in reference [8].

\section{Nonlinear Buckling}

The derivation of the relation between $P_{N L} / P_{L}$, where $P_{N L}$ is the post buckling axial load and $P_{L}$ is the linear buckling load of the hinged beam(column), is more or less goes on the similar lines as present for nonlinear free vibrations, but for the use of the expression for the work done ' $\mathrm{W}$ ' by the induced compressive load ' $\mathrm{p}$ ' in the beam due to uniform heating, in the place of the kinetic energy term ' $\mathrm{T}$ ' in equation(8), and the expression for ' $\mathrm{W}$ '

$$
W=\frac{P}{2} \int_{0}^{L} w_{x}^{2} d x
$$

Following the procedure given in the previous section and minimizing the total potential (U-W) with respect to 'b', the expression for $P_{N L} / P_{L}$ is obtained as

$$
P_{N L} / P_{L}=\left[1+\frac{1}{4}\left(\frac{b}{r}\right)^{2}\right]
$$

As there is no complexity like harmonic or non harmonic oscillations assumption exists for the nonlinear buckling problem, the derivation of the expressions for $P_{N L} / P_{L}$ can be shaped at the stage where equation (9) is obtained for the nonlinear free vibration problem and further refinement of the solution does not exist for the nonlinear buckling problem of beams. 


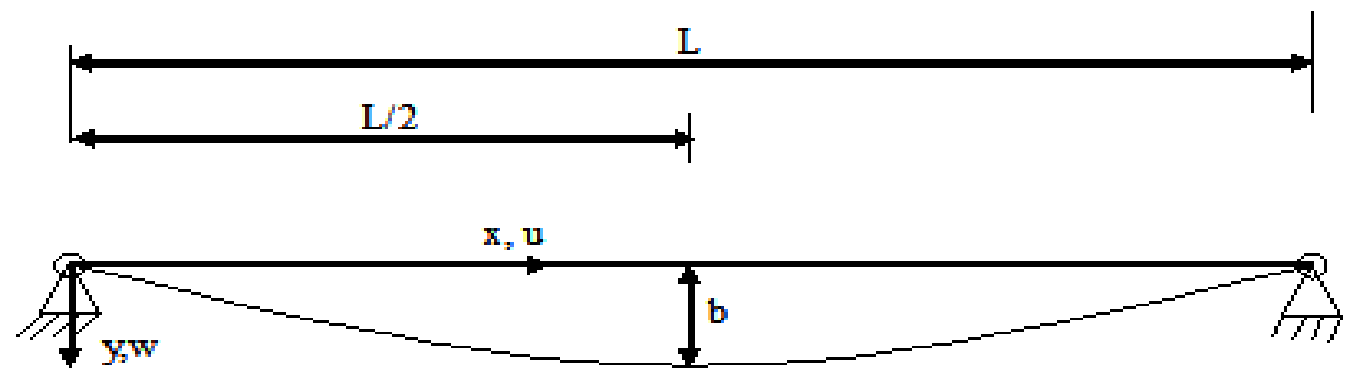

Fig.1. Nonlinear vibration and Buckling of a Hinged Beam

\section{Conclusion}

The improvisations used in the present study for obtaining the nonlinear free vibrations and buckling of hinged beams simplifies the solution procedure substantially and provides accurate solutions. Because of the generality of the improvisations used, these can be effectively applied to obtain simplified quick and accurate solution for the structural elements other then beams like plates and vessels. However, it may be noted that some minor modifications may be necessary for boundary conditions other than hinged ones considered in present work.

\section{Acknowledgements}

The author is highly thankful to the authorities of Sreenidhi Institute of Science \& Technology for their encouragement and support during the course of this work.

\section{References}

[1]. S.Woinowsky - Krieger, "The effect of an axial force on the vibration of hinged bars", Vol.17, 1950, pp.35-36.

[2]. A.V.Srinivasam, "Large amplitude free vibration of beams and plates", AIAA Journal, Vol.3, 1965, pp.1951-53.

[3]. G.Singh, A.K.Sharma and G.V.Rao, "Large-amplitude free vibrations of beams" - A discussion on various formulations and Assumptions, journal of sound and vibration, Vol.142, 1990, pp.77-85

[4]. C.L.Dym, "stability theory and its applications to structural mechanics"Noordhoff international,1974,Leyden, The Netherlands.

[5]. J.M.T.Thompson and G.W.Hunt, "A general theory of elastic stability",1973,Wiley,London.

[6]. G.V.Rao and K.K.Raju, "Thermal post buckling of columns", AIAA Journal, Vol.40, 2001, pp.2138-2140.

[7]. Gwo-Bao Min and J G Eisley "Nonlinear Vibration of Buckled Beams" journal of manufacturing science.Vol.92,2010,pp.637-645.

[8]. A.Barari, H.D. Kaliji, M. Ghadimi; G. Domairry "Non-linear vibration of Euler-Bernoulli beams" Latin American Journal of solids and structures,Vol.8,2011.

[9]. A F E L -Bassiouny ,"Nonlinear vibration of a post-buckled beam subjected to external and parametric excitations" Physica Scripta, Royal academia of sciences, Vol.74,2006.

[10]. H,M.Sedigh, K.H.Shiraj, A.R.Noghrehabadi and A.Hildirim," Asymptotic investigation of buckled beam nonlinear vibration" Transactions of Mechanical Engineering, Vol. 36, No. M2, pp. 107-116. 